\title{
Advanced Stage Hepatocellular Carcinoma Successfully Treated with Liver-directed Concurrent Chemoradiotherapy and Sequential Trans- arterial Radio-embolization
}

\author{
Minho Noh', Beom Kyung Kim ${ }^{1,2,3}$, Seung Up Kim¹,2,3 \\ ${ }^{1}$ Department of Internal Medicine, ${ }^{2}$ Institute of Gastroenterology, Yonsei University College of Medicine, ${ }^{3}$ Yonsei Liver Center, Severance \\ Hospital, Seoul, Korea
}

Received Feb. 9, 2021

Revised Mar. 12, 2021

Accepted Mar. 15, 2021
Optimal treatment strategies for patients with advanced hepatocellular carcinoma (HCC) is yet to be determined. Herein, we present a case of advanced HCC with tumor invasion into the right anterior portal vein and right hepatic vein where complete response (CR) was achieved via a multidisciplinary approach. This patient had a $10.5 \mathrm{~cm}$-sized HCC invading segment $\mathrm{VI}$, without extrahepatic spread. Liver function was classified as Child-Pugh class A, and the performance status was good. Transarterial radio-embolization (TARE) was performed 6 weeks after the completion of liver-directed concurrent chemoradiotherapy, and CR was confirmed 3 months post-TARE. Adoptive cell therapies were performed as adjuvant therapy and CR was maintained for over 15 months, until the local recurrence of a $2 \mathrm{~cm}$-sized HCC was found. Therefore, in selected cases with preserved liver function, combination therapies, including LRTs and systemic therapy, can be a useful therapeutic option for advanced HCC. (J Liver Cancer 2021;21:97-103)

Keywords: Hepatocellular carcinoma; Concurrent chemoradiotherapy; Radioembolization; Yttrium-90

\section{INTRODUCTION}

Approximately one-third of patients with hepatocellular carcinoma (HCC) are diagnosed at an advanced stage with major portal vein (PV) tumor invasion. ${ }^{1}$ The expected prognosis in such cases is typically poor despite the administration of optimal systemic therapy according to the practice guidelines. ${ }^{2}$ Sorafenib or lenvatinib is now an approved oral

\section{Corresponding author : Beom Kyung Kim}

Department of Internal Medicine, Yonsei University College of Medicine, 50-1 Yonsei-ro, Seodaemun-gu, Seoul 03722, Korea

Tel. +82-2-2227-4184, Fax. +82-2-393-6884

E-mail; beomkkim@yuhs.ac

https://orcid.org/0000-0002-5363-2496 multi-kinase inhibitor for prolonging the overall survival (OS) of patients with advanced-stage HCC. However, data supporting the survival benefits of molecular-targeted agents among patients with advanced-stage HCC with major PV invasion are still limited. ${ }^{3}$

Therefore, diverse treatment approaches, including a combination of various loco-regional therapies (LRT) and/ or systemic treatments, including transarterial chemoembolization (TACE) plus radiotherapy (RT), tremelimumab with ablation, liver-directed concurrent chemoradiotherapy (LDCCRT) plus sequential sorafenib, and transarterial radioembolization (TARE) in combination with sorafenib, have been attempted in a concurrent or sequential manner. ${ }^{4-8}$ Among these, TARE with Yttrium-90 (Y-90) microspheres 
has gained increasing acceptance as an alternative to TACE over the past decade and has become an increasingly popular treatment option owing to an increased and durable response and better tolerability. ${ }^{9}$ However, TARE is contraindicated in patients with an excessive lung shunt fraction (LSF). ${ }^{10}$ Herein, we report a case of advanced-stage HCC in which a tumor with high LSF was initially treated with LD-CCRT, which was subsequently delivered after confirmation of decreased lung shunting, leading to a complete response.

\section{CASE REPORT}

\section{Clinical finding}

A 55-year-old man presented with a large liver mass incidentally detected on ultrasonography screening at another hospital. Although he uses to consume 2-3 bottles of beer per week, he did not have any underlying liver disease. There were no associated symptoms or remarkable findings on physical examination. The initial laboratory findings were as follows: white blood cell count, 5,670/ $\mu \mathrm{L}$; hemoglobin, $14.5 \mathrm{~g} / \mathrm{dL}$; platelet count, $175,000 / \mu \mathrm{L}$; total bilirubin, $0.9 \mathrm{mg} /$ $\mathrm{dL}$; albumin, $4.9 \mathrm{~g} / \mathrm{dL}$; prothrombin time-international normalized ratio, 1.09; aspartate aminotransferase, $142 \mathrm{U} / \mathrm{L}$; and alanine aminotransferase, 157 U/L. Both hepatitis B surface antigen and anti-hepatitis $\mathrm{C}$ virus antibody were negative. The level of alpha-fetoprotein (AFP) was 4,868 ng/mL, and protein induced by the absence of vitamin $\mathrm{K}$ or antagonist-II (PIVKA-II) level was 27,544 mAU/mL. Liver function was preserved with a Child-Pugh score of 5 and the Eastern Cooperative Oncology Group performance status was 1.

\section{Imaging findings}

Initial liver dynamic magnetic resonance imaging (MRI) revealed a $10.5 \mathrm{~cm}$ large tumor diagnosed as HCC with typical radiological features including hypervascularity in the arterial phase and washout in the portal and delayed phases, at
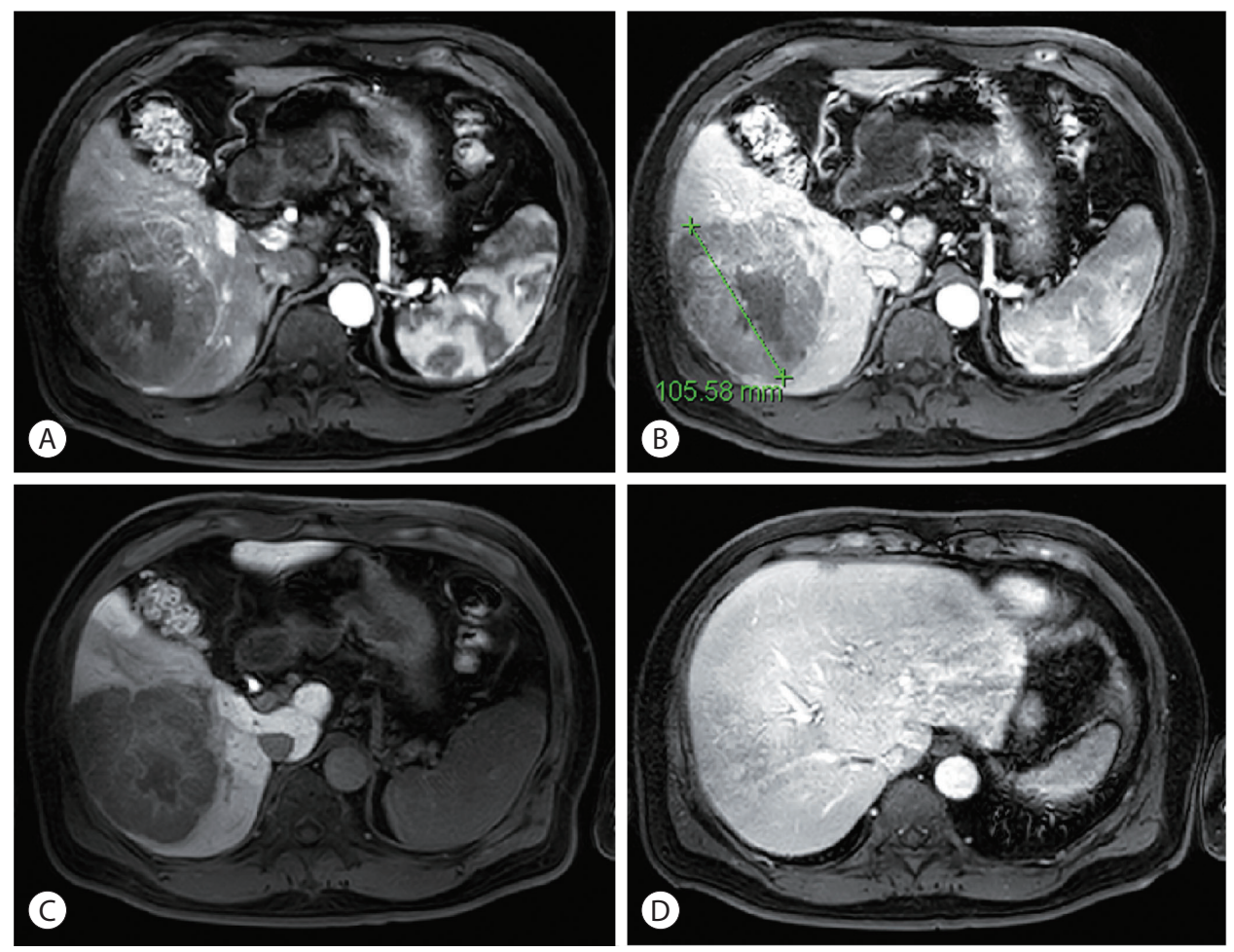

Figure 1. Initial liver dynamic magnetic resonance imaging findings. A single $10.5 \mathrm{~cm}$-sized mass in segment VI showed enhancement in the arterial phase (A), and washout in the portal (B) and delayed phase (C). Tumor thrombosis was seen in the right hepatic vein and right anterior portal vein (D). 
segment S6 (Fig. 1). Tumor thrombosis was observed in the right hepatic vein and the right anterior PV. Chest computed tomography (CT) revealed no evidence of hematogenous lung metastasis or lymph node enlargement.

\section{Diagnosis and treatment}

The patient was diagnosed with advanced stage HCC (BCLC, stage C; modified Union for International Cancer Control T3N0M0, stage III) without liver biopsy, based on typical imaging findings. First, we planned TARE with Yttrium-90; however, lung scintigraphy using technetium-99mlabeled albumin macroaggregates (MAA-99mTc) revealed an intense radiotracer in the bilateral lung field, with an LSF of $61.59 \%$ (Fig. 2A). Since the patient was at high risk of excessive pulmonary irradiation after TARE, we planned LDCCRT as an alternative. The major aims of LD-CCRT were to reduce not only the tumor burden but also the LSF.

A total of 75 Gy was prescribed in 25 fractions of $3.0 \mathrm{~Gy}$ over 5 weeks using a 10-megavolt linear accelerator. It was intended to deliver $95 \%$ of the prescribed dose encompassing the planning target volume around the clinical target volume. Concurrent continuous-infusion hepatic arterial 5-fluorouracil (at a dose of $500 \mathrm{mg} /$ day) was delivered during the first and fifth weeks of RT through a percutaneous hepatic arterial catheter (Chemoport), implanted at the time of initial hepatic arterial angiography.

Three weeks after the completion of LD-CCRT, a response evaluation using CT was performed, which showed that the main tumor size had decreased from $10.5 \mathrm{~cm}$ to $8 \mathrm{~cm}$ and right PV thrombosis was not obvious (Fig. 3A). To determine whether TARE is eligible as a subsequent treatment modality, technetium-99m labeled MAA was employed again, revealing a markedly decreased LSF of 12.15\% (Fig. 2B). Thus, based on a multi-disciplinary approach, TARE was subsequently performed to treat the remnant tumor 6 weeks after the completion of LD-CCRT. SIR-sphere ${ }^{\circledR}$ Y-90 resin microspheres (Sirtex Medical, North Sydney, Australia) with a total infused radiation activity of 2.5 gigabecquerel was infused via the right hepatic artery. The calculated lung dose was 17.9.

Post-treatment CT and MRI were performed sequentially (Fig. 3). AFP and PIVKA-II had decreased to normal levels. Both radiologic and biological CR was confirmed at 3 months post-TARE. As an adjuvant therapy, adoptive cell transfer (ACT) therapy using Immunecell-LC ${ }^{\circledR}$ (Green cross cell, Yongin, Korea) injection of $\left(1.0 \times 10^{8}\right)-\left(2.0 \times 10^{9}\right)$ autologous cytokine induced killer cells was performed 7 times in the duration of 10 weeks.

Ten days after the 7th ACT (19 weeks after TARE), the pa-
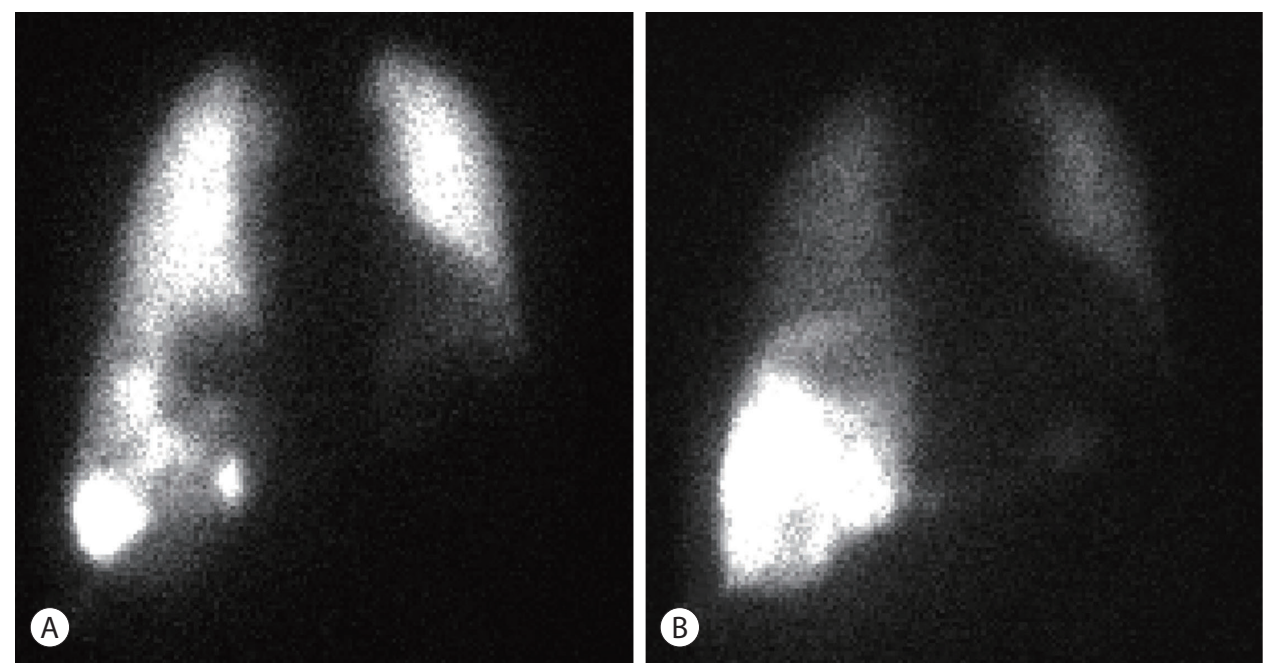

Figure 2. Technetium-macroaggregated albumin scintigram of the patient revealed $61.59 \%$ lung shunt fraction (LSF), before liver-directed concurrent chemoradiotherapy (LD-CCRT) (A). Five weeks after LD-CCRT, LSF was decreased to $12.15 \%$ (B). 
tient visited the emergency department with complains of dyspnea. On physical examination, the patient presented with tachypnea and bilateral crackles on pulmonary auscul- tation, with no signs of heart failure. Chest CT revealed consolidations in both lungs with right pleural effusion, consistent with radiation pneumonitis (Fig. 4A). Treatment began
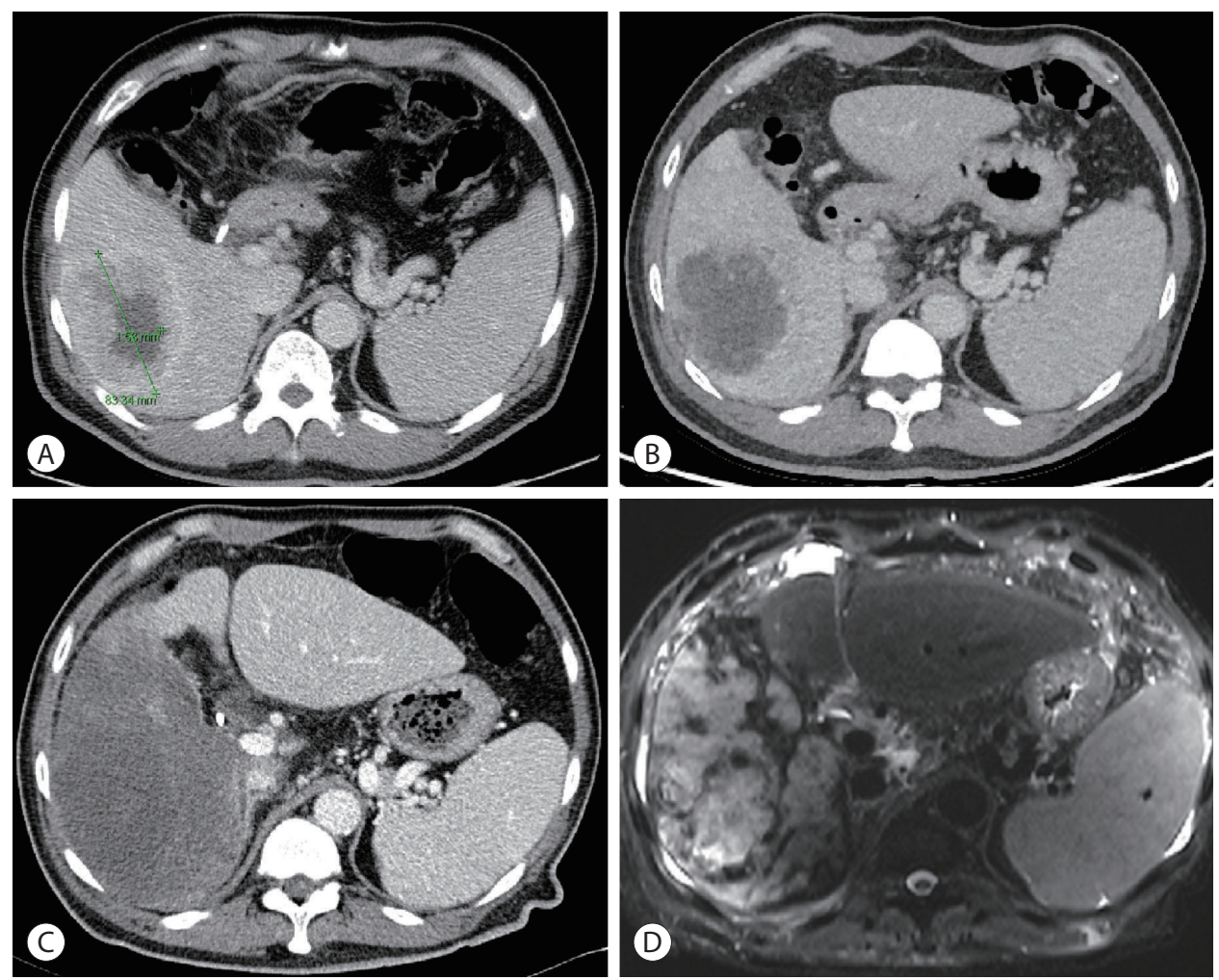

Figure 3. Follow-up liver dynamic computed tomography (CT) and magnetic resonance imaging (MRI) findings. (A) CT at delayed phase, 3-weeks post-liver-directed concurrent chemoradiotherapy: decreased size of the mass in the right lobe with right hepatic vein thrombosis, from $10.5 \mathrm{~cm}$ to $8 \mathrm{~cm}$. (B) CT at delayed phase, 3-weeks post-transarterial radio-embolization (TARE): markedly decreased tumor vascularity without remarkable change of overall size of the treated tumor in the right posterior liver, $7.6 \mathrm{~cm}$. Diffuse parenchymal atrophy of the right liver observed. (C) CT at delayed phase, 6-weeks post-TARE: change in post-treatment infarction and markedly decreased attenuation of the right hemiliver. (D) T2 weighted MRI image, 3-months post-TARE: total necrosis of the right liver with focal capsular disruption.
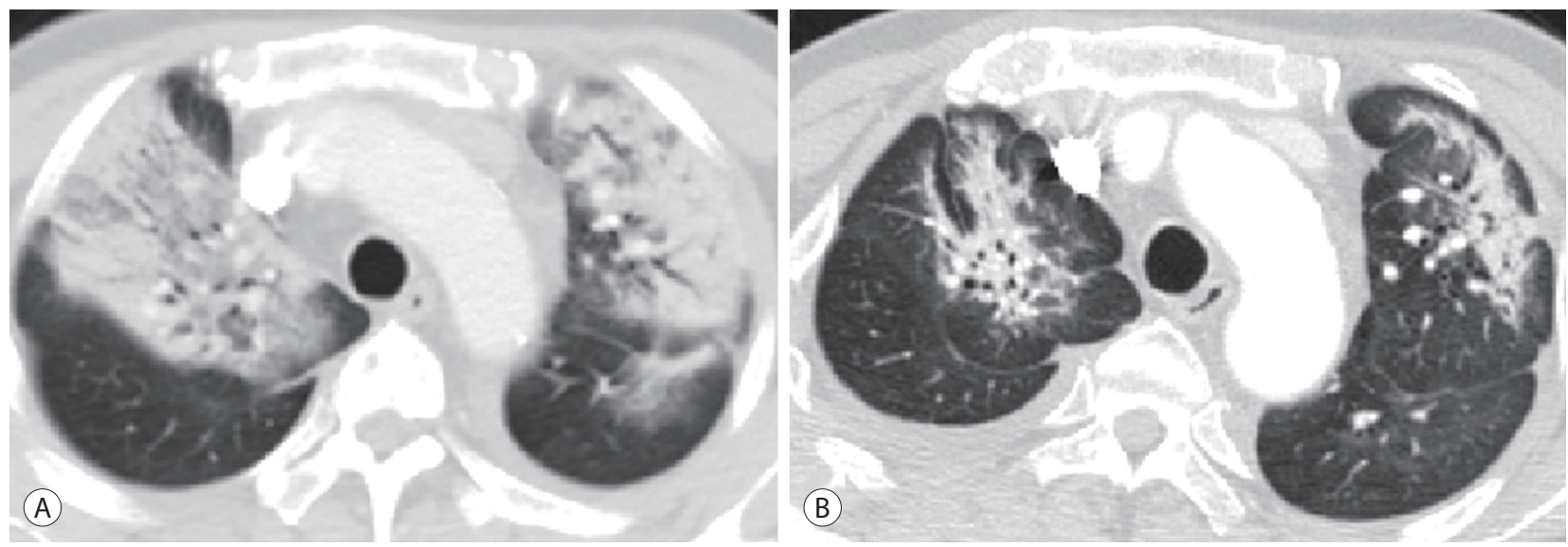

Figure 4. Chest high-resolution computed tomography images showing bilateral ground glass opacities and interlobular septal thickening at 19-weeks post-transarterial radio-embolization (A), remnant radiation pneumonitis after 4 months of steroid therapy (B). 
with $125 \mathrm{mg}$ /day of methylprednisolone with a total tapering duration of 4 months. The patient's clinical condition improved with the considerable resolution of radiation pneumonitis on CT that was taken 4 months after steroid therapy (Fig. 4B). The patient exhibited remarkable pulmonary parenchymal recovery, with no evidence of pulmonary dysfunction or the need for oxygen.

Fifteen months after CR, follow-up CT showed an approximately $2 \mathrm{~cm}$ recurrent tumor with washout in the delayed phase at liver S7 (Fig. 5A). TACE using $20 \mathrm{mg}$ of adriamycin and lipiodol mixture was performed, and compact lipiodol uptake in the recurrent tumor at liver S7 was found at 3 weeks post-TACE CT, without new or residual tumor (Fig. 5B). The patient remains alive with no remnant or recurrent tumors in the liver. The clinical course is described in Fig. 6 according to the treatment modalities and changes in tumor markers.

\section{DISCUSSION}

In this study, we present a case of a large single HCC treated with two types of LRTs, including LD-CCRT and TARE.
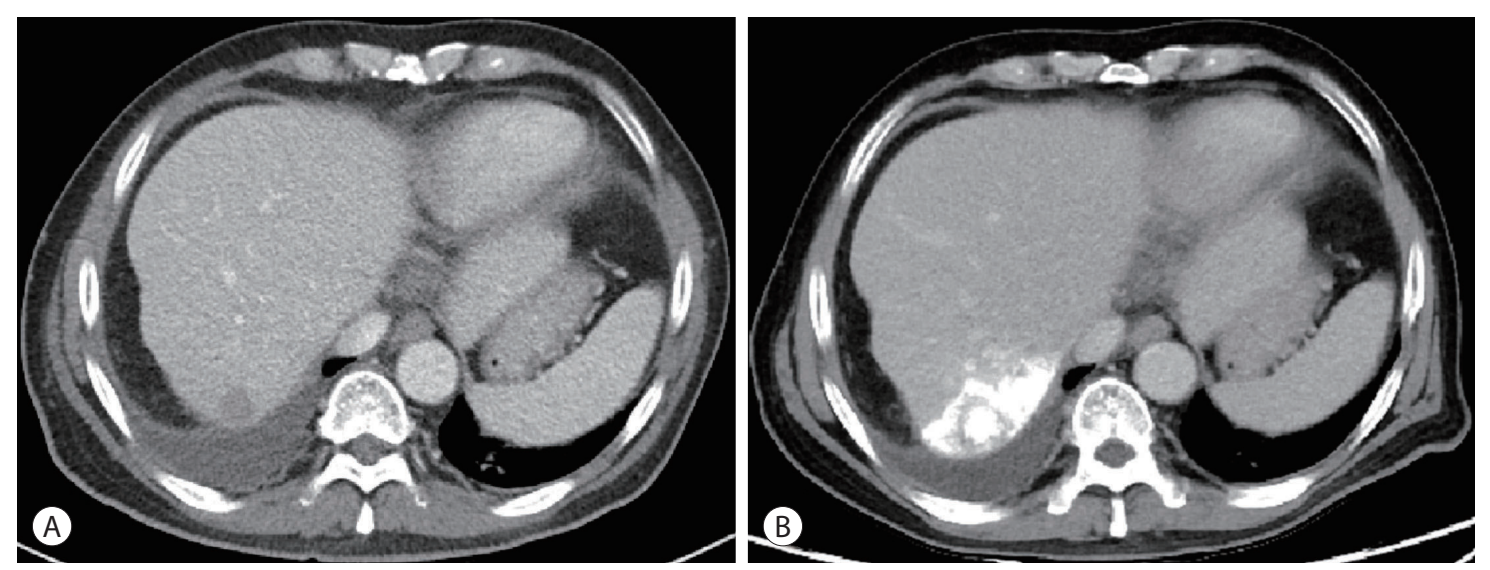

Figure 5. Findings on liver dynamic computed tomography images. Newly noted recurrent tumor at liver S7, about $2 \mathrm{~cm}$ at 19 -months posttransarterial radio-embolization (A). Compact lipiodol uptake in the recurrent tumor at liver S7 without new or residual tumor, 1-month posttransarterial chemoembolization (B).

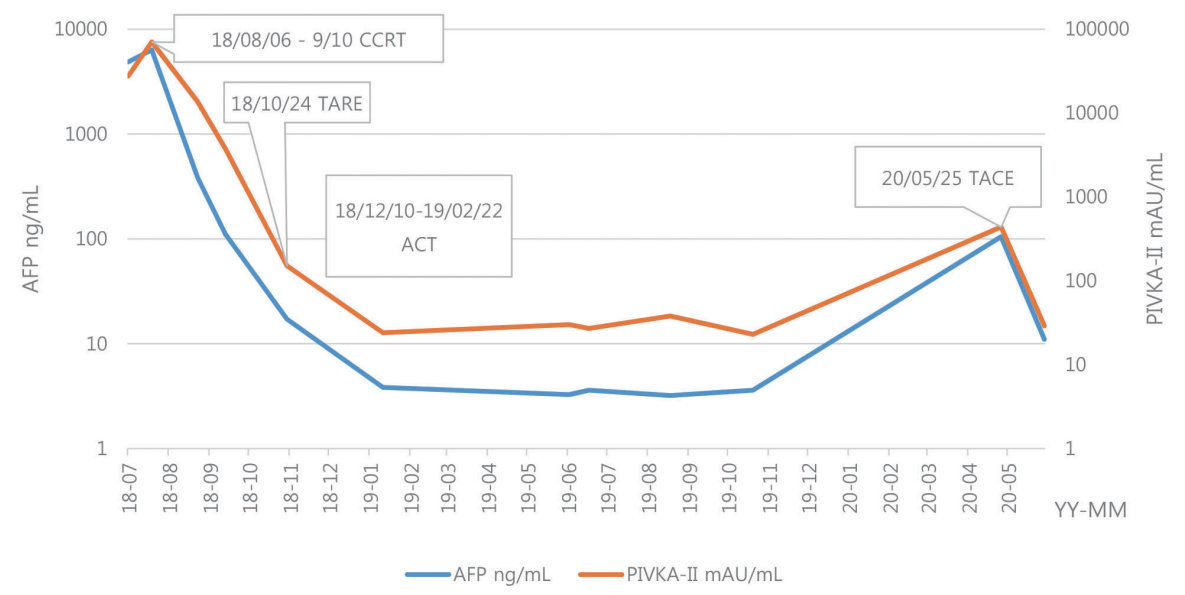

Figure 6. Cinical course of the patient with alpha-fetoprotein (AFP), prothrombin induced by vitamin K absence (PIVKA)-II. A biological complete response was achieved at 3-weeks post-transarterial radio-embolization. CCRT, concurrent chemoradiotherapy; TARE, transarterial radioembolization; ACT, adoptive cell transfer; TACE, transarterial chemoembolization. 
We did not consider surgical resection as a treatment option because both high serum AFP levels and major vascular invasion are major predictors of poor survival after surgical resection in patients. ${ }^{11,12}$

According to current practice guidelines, systemic sorafenib or lenvatinib is the first-line therapeutic option for advanced HCC. ${ }^{5}$ However, the present patient had neither underlying hepatitis nor cirrhosis, and exhibited preserved liver function; we therefore thought that palliative treatment alone might be a suboptimal option for the patient. We considered TARE as the first option of LRT. Some studies have suggested that TARE is more advantageous than sorafenib in PVT. Edeline et al. ${ }^{13}$ showed a median OS of 26.2 versus 8.7 months in patients treated with TARE versus sorafenib. However, TARE is contraindicated in patients with a high LSF, primarily due to radiation-induced lung injury. ${ }^{10}$ Considering that $20 \%$ of LSF is the generally accepted limit of SIR-Spheres, our patient could not be a candidate for TARE owing to a high LSF of $61.59 \%$.

To date, there have been several studies to manage high LSF in patients undergoing TARE, including bland embolization or chemoembolization, hepatic vein occlusion balloons, low-dose radio-embolization, and chemotherapy. ${ }^{14,15}$ One of suggested mechanisms about chemotherapy is that "normalizing" the tumor vascularity with chemotherapy not only reduces the abnormal vessels including shunts but also increases blood flow to the tumors, making tumor more responsive to TARE. ${ }^{14}$ Since LD-CCRT includes both local RT and concurrent hepatic arterial infusion chemotherapy, LSF might be effectively decreased. Furthermore, tumor shrinkage through LD-CCRT has also been reported, which could prolong survival in patients with advanced but liver-confined HCC. ${ }^{16}$ LD-CCRT may be adopted as a proper option in this case.

Liver dynamic CT at 3 weeks after LD-CCRT showed that the mass had decreased from $10.5 \mathrm{~cm}$ to $8 \mathrm{~cm}$. We excluded surgical resection as a subsequent treatment option because PV thrombosis could not be excluded on follow-up CT. Regarding TARE, lung scintigraphy was performed again, which showed a $12.15 \%$ LSF. TARE was performed 1 week after lung scintigraphy because delaying TARE could increase the LSF.

Despite a reduced LSF, radiation pneumonitis occurred 19 weeks post-TARE. Radiation pneumonitis often occurs as a post-TARE complication because the lung tissue is susceptible to radiation damage. ${ }^{17}$ Leung et al. ${ }^{17}$ reported that 55.6\% (5 out of 9) of patients with lung shunting greater than $13 \%$ developed radiation pneumonitis. Even though the LSF was reduced to $12.15 \%$ by $\mathrm{LD}$-CCRT, there is a possibility that new collaterals and arteriovenous shunts opened up between lung scintigraphy and TARE. Our patient was able to return to his daily life after 4 months of steroid therapy.

We found an optimal response on CT and planned consecutive systemic therapy. So far, there has been no proven benefit for molecular target agents such as sorafenib to reduce recurrence after achievement of $C R{ }^{18}$ Meanwhile, additional immunotherapeutic approaches, such as ACT, have been actively researched as a combination modality with other systemic therapies or LRT. ${ }^{19}$ ACT improves the quality and quantity of anti-cancer cells and reduces the immune system's tolerance to cancer cells. Previous studies have shown that adoptive immunotherapy could lower the frequency of recurrence and improve recurrence-free outcomes after surgery or radiofrequency ablation for HCC. ${ }^{20}$ ATC therapy was the appropriate option in this case. Although a $2 \mathrm{~cm}$-sized HCC had recurred once, the patient had been in a healthy state without HCC until 30 months after diagnosis.

In conclusion, we described a case of advanced-stage HCC that was successfully treated with a combination therapy of LRT and adjuvant systemic therapy. Thus, LD-CCRT and sequential TARE may be a useful treatment option for selected patients with advanced-stage HCC. Although current guidelines for advanced HCC still favor systemic agents as a standard of care, various studies on the role of active LRTs have been in progress for advanced HCC with preserved liver function and without extrahepatic involvement. Further research for sub-classification of advanced-stage HCC, where active LRTs would be more advantageous than systemic therapy alone is necessary.

\section{Conflicts of Interest}

The authors declare no competing interests. 


\section{REFERENCES}

1. Chan SL, Chong CCN, Chan AWH, Poon DMC, Chok KS. Management of hepatocellular carcinoma with portal vein tumor thrombosis: review and update at 2016. World J Gastroenterol 2016:22:7289-7300.

2. Díaz-González Á, Reig M, Bruix J. Treatment of hepatocellular carcinoma. Dig Dis 2016;34:597-602.

3. Moriguchi M, Aramaki T, Nishiofuku H, Sato R, Asakura K, Yamaguchi $K$, et al. Sorafenib versus hepatic arterial infusion chemotherapy as initial treatment for hepatocellular carcinoma with advanced portal vein tumor thrombosis. Liver Cancer 2017;6:275286.

4. Yoon SM, Ryoo BY, Lee SJ, Kim JH, Shin JH, An JH, et al. Efficacy and safety of transarterial chemoembolization plus external beam radiotherapy vs sorafenib in hepatocellular carcinoma with macroscopic vascular invasion: a randomized clinical trial. JAMA Oncol 2018;4:661-669.

5. Duffy AG, Ulahannan SV, Makorova-Rusher O, Rahma O, Wedemeyer $H$, Pratt $D$, et al. Tremelimumab in combination with ablation in patients with advanced hepatocellular carcinoma. J Hepatol 2017;66:545-551.

6. Colagrande S, Inghilesi AL, Aburas S, Taliani GG, Nardi C, Marra F. Challenges of advanced hepatocellular carcinoma. World J Gastroenterol 2016;22:7645-7659.

7. Kim BK, Kim DY, Byun HK, Choi HJ, Beom SH, Lee HW, et al. Efficacy and safety of liver-directed concurrent chemoradiotherapy and sequential sorafenib for advanced hepatocellular carcinoma: a prospective phase 2 trial. Int J Radiat Oncol Biol Phys 2020;107:106-115.

8. Han S, Lee HW, Park JY, Kim SU, Kim DY, Ahn SH, et al. Appraisal of long-term outcomes of liver-directed concurrent chemoradiotherapy for hepatocellular carcinoma with major portal vein invasion. J Hepatocell Carcinoma 2020;7:403-412.

9. Salem R, Gabr A, Riaz A, Mora R, Ali R, Abecassis M, et al. Institutional decision to adopt $Y 90$ as primary treatment for hepatocellular carcinoma informed by a 1,000-patient 15-year experience. Hepatology 2018;68:1429-1440.
10. Sangro B, Gardini AC. Radioembolisation in hepatocellular carcinoma: principles of management. In: Cross T, Palmer DH, eds. Liver cancers: from mechanisms to management. Cham: Springer International Publishing, 2019:139-152.

11. Pawlik TM, Poon RT, Abdalla EK, Zorzi D, Ikai I, Curley SA, et al. Critical appraisal of the clinical and pathologic predictors of survival after resection of large hepatocellular carcinoma. Arch Surg 2005;140:450-457; discussion 457-458.

12. Hermanek $P$, Hendson DE, Hutter RVP, Sobin LH. TNM supplement 1993: a commentary on uniform use. Heidelberg: Springer-Verlag; 1993:1-142.

13. Edeline J, Crouzet L, Campillo-Gimenez B, Rolland Y, Pracht M, Guillygomarc'h A, et al. Selective internal radiation therapy compared with sorafenib for hepatocellular carcinoma with portal vein thrombosis. Eur J Nucl Med Mol Imaging 2016;43:635-643.

14. Schiro BJ, Amour ES, Harnain C, Gandhi RT. Management of high hepatopulmonary shunts in the setting of $Y 90$ radioembolization. Tech Vasc Interv Radiol 2019;22:58-62.

15. Theysohn JM, Schlaak JF, Müller S, Ertle J, Schlosser TW, Bockisch $A$, et al. Selective internal radiation therapy of hepatocellular carcinoma: potential hepatopulmonary shunt reduction after sorafenib administration. J Vasc Interv Radiol 2012;23:949-952.

16. Cha H, Yoon HI, Lee IJ, Koom WS, Han KH, Seong J. Clinical factors related to recurrence after hepatic arterial concurrent chemoradiotherapy for advanced but liver-confined hepatocellular carcinoma. J Radiat Res 2013;54:1069-1077.

17. Leung TW, Lau WY, Ho SK, Ward SC, Chow JH, Chan MS, et al. Radiation pneumonitis after selective internal radiation treatment with intraarterial 90Yttrium-microspheres for inoperable hepatic tumors. Int J Radiat Oncol Biol Phys 1995;33:919-924.

18. Mokdad AA, Singal AG, Yopp AC. Advances in local and systemic therapies for hepatocellular cancer. Curr Oncol Rep 2016;18:9.

19. Hendrickson PG, Olson M, Luetkens T, Weston S, Han T, Atanackovic $D$, et al. The promise of adoptive cellular immunotherapies in hepatocellular carcinoma. Oncoimmunology 2020;9:1673129.

20. Takayama T, Sekine T, Makuuchi M, Yamasaki S, Kosuge T, Yamamoto J, et al. Adoptive immunotherapy to lower postsurgical recurrence rates of hepatocellular carcinoma: a randomised trial. Lancet 2000;356:802-807. 